J. Clin. Chem. Clin. Biochem.

Vol. 15, 1977, pp. 473-478

\title{
Kreuzimmunelektrophoresen auf Celluloseacetatfolie
}

Von K.-H. Rehfeld

Zentrallaboratorium des Krankenhauses Am Urban (Chefarzt: Dr. W. Herold), Krankenhausbetrieb von BerlinKreuzberg

(Eingegangen am 10. Januar/31. März 1977)

Zusammenfassung: Es wird eine einfache Technik für Kreuzimmunelektrophoresen auf Celluloseacetatfolie und eine Methode zur Identifizierung der Protein-Peaks mit monospezifischen Antiseren beschrieben.

\section{Crossed immunoelectrophoresis on cellulose acetate}

Summary: A simple technique for crossed immunoelectrophoresis on cellulose acetate membranes and a method for the identification of the protein peaks with monospecific antisera is described.

\section{Einleitung}

Mit der nunmehr klassischen, qualitativen Immunelektrophorese (1) sind quantitative Aussagen über die Konzentration der dargestellten Proteine nur mit großen Einschränkungen zu treffen (2). Quantitative Aussagen wurden erst mit der Einführung der Kreuzimmunelektrophorese (CIEF) möglich, bei der das elektrophoretisch getrennte Material (1. Dimension) um $90^{\circ}$ gedreht während einer zweiten Elektrophorese (2. Dimension) in ein antikörperhaltiges Medium einwandert. Die einzelnen Proteine bilden dabei Präzipitate in der Form von Gauß-Glockenkurven, deren Fläche $(3,4)$ bzw. bei Anwendung einer besonderen Formel $(5)$ auch deren Höhe direkt proportional der Konzentration des Antigens bzw. umgekehrt proportional der Konzentration des korrespondierenden Antiköṛpers ịst.

Seit den grundlegenden Arbeiten von Ressler (6), Laurell (7), Freeman \& Clarke (3) hat die Technik der Kreuzimmunelektrophorese zahlreiche Modifikationen erfahren ((8-23) Literaturübersicht: (24)). Nahezu in allen diesen Arbeiten wurde Agar oder Agarose als Trägermedium verwwendet. Nach Kenntnis der Literatur wurde nur in żwei Fällen versucht, Kreuzimmunelektrophorese auf Trägerfolien durchzuführen, wobei kunststoff verstärkte Celluloseacetatfolien (17) bzw. żusätzlich gelatinierte Folien (18) verwendet wurden. Nachdem in unserem Hause seịt einigen Jahren routinemäßig qualitative Immunelektrophoresen auf Celluloseacetatfolie durchgeführt werden (25), wurde eine einfache
Technik erarbeitet, die es erlaubt, quantitative Immunelektrophoresen (Kreuzimmunelektrophoresen) mit dem gleichen Material anzufertigen.

\section{Material und Methode}

Die Trennungen der ersten und zweiten Dimension der Kreuzimmunelektrophorese erfolgten auf zwei getrennten Folien. Dabei erfolgte in der zweiten Dimension eine Wanderung der Proteine von der senkrecht gestellten ersten Folie über deren Längskante auf die waagerecht aufgelegte zweite, antiserumhaltige Folie.

Der Kammerpuffer für beide Trennungen wurde aus einer fertig bezogenen Puffersubstanz $z^{1}$ ) durch Lösen einer Packung in 21 bidest. Wasser hergestellt (pH 8,6; Molarität 0,05; Herstellerangaben).

Das in allen Versuchen verwendete Poolserum (Gesamteiweiß $7,5 \mathrm{~g} / \mathrm{dl}$, Biuret) wurde aus Seren von 20 Patienten mit normaler Zonenelektrophorese hergestellt und in kleinen Portionen bei $-20^{\circ} \mathrm{C}$ aufbewahrt. Für die Trennung wurde es $1+30$ mit Kammerpuffer verdünnt und mit einem Mikrodispenser ${ }^{2}$ ) $2 \mu \mathrm{l}$ je Folie aufgetragen.

Als Netzgerät diente das Elektrophorese Netzgerät FB II der Fa. Bergmann, Berlin.

\section{Dimension}

Vier Celluloseacetatfolien des Streifenformats $145 \times 25,5 \mathrm{~mm}^{1}$ ) wurden mit entsprechendem Kugelschreiber wie in Abbildung 1 ersichtlich markiert und ggf. numeriert. Nach dem Einweichen in Kammerpuffer für 5-10 min wurden sie möglichst gleichmäßig zwischen angefeuchtetem Filterpapier abgetrocknet, in

1) Fa. Sartorius, Göttingen

2) Fa. Behring, Marburg 


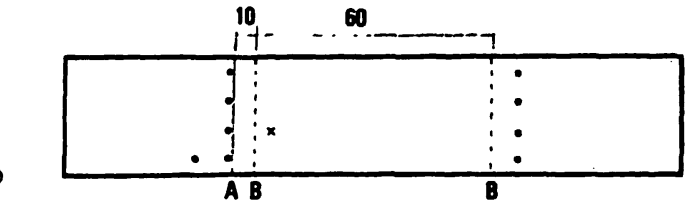

Abb. 1. Markierungen auf den Folien der 1. Dimension. Maße in Millimeter. A: Hilfslinie an der kathodischen Perforation für die weiteren Abmessungen. Die unterbrochenen Linien B kommen bei der Trennung der 2. Dimension in die Schlitze der Haltevorrichtung. X: Auftragstelle, jeweils $10 \mathrm{~mm}$ von der kathodischen Perforation bzw. vom seitlichen Rand entfernt. die Haltevorrichtungen ${ }^{3}$ ) gespannt und in die Elektrophoresekammer ${ }^{3}$ ) gebracht. Nach dem Materialauftrag erfolgte die Trennung der ersten Dimension mit konstant $250 \mathrm{~V} / \mathrm{Kammer}$ während 40 Minuten bei Raumtemperatur.

\section{Dimension}

Aus einer Celluloseacetatfolie des Formats $145 \times 192 \mathrm{~mm}^{1}$ ) wurden vier Folien $56 \times 90 \mathrm{~mm}$ ausgeschnitten und numeriert.

$\oplus \quad$ Für vier Folien wurden $200 \mu \mathrm{l}$ polyvalentes Anthihumanserum vom Kaninchen ${ }^{4}$ ) mit $2250 \mu \mathrm{l}$ Kammerpuffer verdünnt. Pro Folie wurden $600 \mu \mathrm{l}$ dieser Mischung auf den glatten Boden einer rostfreien Metallschale $(22 \times 15 \times 5 \mathrm{~cm})$ mit dicht schließendem Deckel gegeben, wobei die AntihumanserumPuffer-Depots so angeordnet wurden, daß die vier anschließend darauf flach aufgelegten Celluloseacetatfolien $(56 \times 90 \mathrm{~mm})$ weder den Rand der Schale noch sich gegenseitig berührten. Die Folien wurden für 30-40 Minuten in der verschlossenen und waagerecht aufgestellten Schale belassen, um eine gleichmäßige Durchtränkung mit dem Antihumanserum-PufferGemisch zu erreichen.

Die Haltevorrichtungen für die 2. Dimension wurden aus Pertinax hergestellt (Abb. 2). Nach beendeter Trennung in der 1. Dimension wurde zunächst. je eine leere, feuchte Streifenformatfolie $(145 \times 25,5 \mathrm{~mm})$ senkrecht gestellt und
90

.
30
60
60

5.

15

$\theta$

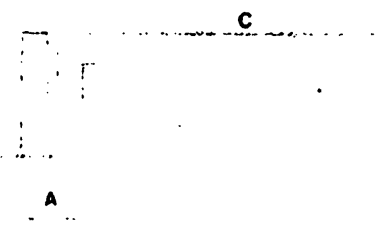

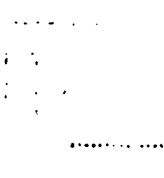

B

Abb. 2. a) Haltevorrichtung für die 2. Dimension. Maße in Millimeter. b) desgl. Seitenansicht mit eingelegten Folien. Bezeichnungen wie Abb. 3.

mit den Längskanten quer zur Laufrichtung der 2. Dimension in die später zur Anode gerichteten Halteschlitze als Verbindung zum anodischen Puffer eingelegt. Anschließend wurden die Folien mit der ersten Trennung (diese nach oben) analog in die gegenüberliegenden Schlitze eingesetzt (Abb. 3a). Die überstehenden Folienenden wurden abgeschnitten. Jede fertig bestückte Haltevorrichtung wurde sofort in die Kammer eingesetzt, um ein Austrocknen der Folien zu vermeiden. Befanden sich alle vier Haltevorrichtungen in der Kammer, wurden die antiserumhaltigen Folien waagerecht so aufgelegt, daß sie auf beiden Seiten auf den Längskanten der Streifenformatfolien auflagen (Abb. 3b). Es wurde Wert darauf gelegt, daß die Folien außerdem nur auf den Stützen in der Mitte der Haltevorrichtungen auflagen und deren Seitenwände sonst nicht berührten, um Flüssigkeitsansammlungen durch Kondensation oder Kohäsion zu vermeiden.

Die Trennung in der 2. Dimension erfolgte bei konstant 40 V/Kammer während 22-23 Stunden bei Raumtemperatur.

Nach beendeter Trennung wurden die aufgelegten Folien entnommen und zur Entfernung nicht präzipitierter Proteine in $400 \mathrm{ml}$ physiologischer $\mathrm{NaCl}$-Lösung 30 Minuten unter Rühren mit einem Magnetrührer ausgewaschen.

Anschließend wurden die Folien 5-10 Minuten gefärbt und fixiert. (Färbe- und Fixationslösung (26): $1 \mathrm{~g}$ Nigrosin ${ }^{5}$ ), $15 \mathrm{~g}$ Trichloressigsäure, $15 \mathrm{~g}$ Salicylsäure, bidest. Wasser ad $500 \mathrm{ml}$ ). Das Auswaschen überschüssiger Farbe erfolgte nach
Sicht in mehreren Bädern mit Essigsäure $(50 \mathrm{~g} / \mathrm{l})$ in etwa 45 Minuten. Die entfärbten Folien wurden zunächst zwischen Filterpapier und danach an der Luft getrocknet.

\section{Messen der Peakhöhen}

Die Folien wurden durch Bestreichen mit flüssigem Paraffin transparent gemacht, in die Negativbühne eines für Negative bis $6 \times 6 \mathrm{~cm}$ ausgerüsteten Vergrößerungsgerätes gelegt und auf hart kopierendes Vergrößerungspapier $(18 \times 24 \mathrm{~cm})$ vergrößert.

Für die Versuche zur Reproduzierbarkeit iv urden identische Peaks mit gleichen Nummern versehen und ihre Höhe über der Basislinie gemessen.

\section{Identifikation}

Zur Identifizierung wurde in einem F-all ein gereinigtes Proteinpräparat (Transferrin, reinst ${ }^{6}$ ) dem Poolserum zugesetzt und damit ein im Vergleich mit reinem Poolserum höherer Peak erzielt (Abb. 6).

3) Fa. Il-Boskamp, Her sel, Miniphor-System

4) Fa. Medac, Hamburg, Code-Nr. $100 \mathrm{SF}$

5) Fa. Merck, Därmstadt

6) Fa. Behring, Marburg

\section{$\oplus$}



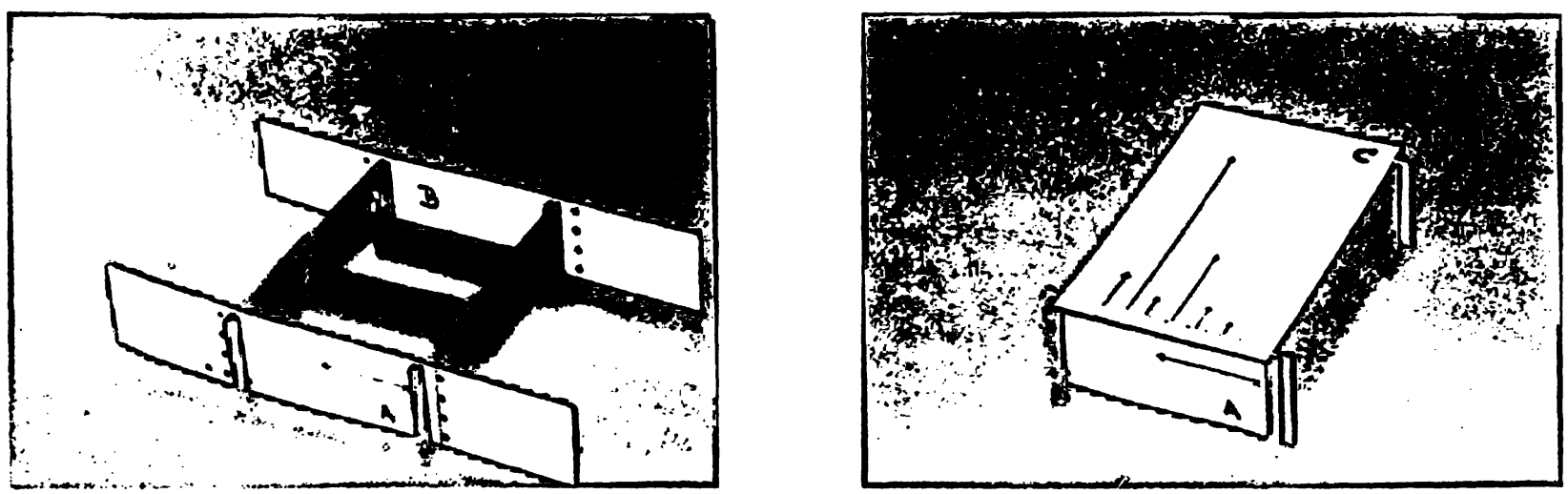

Abb. 3. Haltevorrichtung für die 2. Dimension mit eingelegten Folien (Demonstration).

a) A: Folie mit der Trennung der 1. Dimension (Pfeil). B: leere Folie.

b) Nach dem Abschneiden der überstehenden Folienenden wurde die antiserumhaltige Folie $C$ aufgelegt. Die Pfeile symbolisieren die Trennrichtung der 2. Dimension.

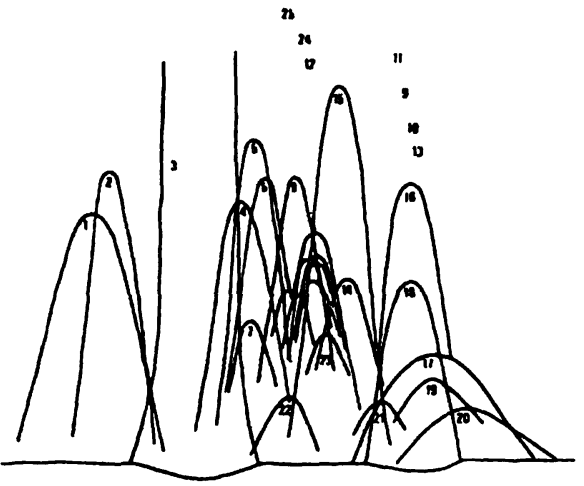

$\oplus$

Abb. 4. Schema einer mit der beschriebenen Methode angefertigten Kreuzimmunelektrophorese (Poolserum). Identifizierte Proteine: $1 \alpha_{1}$-Lipoprotein; 3 Albumin; $4 \alpha_{1}$-Antitrypsin; 9 Ceruloplasmin; $10 \alpha_{2}$-Makroglobulin; 15 Haptoglobin; 18 Transferrin.

Das Albumin wurde aufgrund der elektrophoretischen Beweglichkeit und der Peakhöhe identifiziert.

Einige weitere Proteine wurden mit monospezifischen Antiseren den entsprechenden Präzipitaten zugeordnet.

Wegen des unerwartet hohen Anteils an humanen Ballastproteinen in den handelsüblichen, absorbierten Antiseren, zeigten Kreużimmunelektrophoresen des Poolserums, dem ein monospezifisches Antiserum zugesetzt worden war, ein unübersichtliches Bild mit erhöhten, unregelmäßig geformten Präzipitaten. Einige Peaks traten neu auf.

Um dennoch eine Identifizierung mit monospezifischen Antiseren durchführen zu können, wurde dieses zunächst einer Elektrophorese unterzogen, wobei es auf der Folie der 1. Dimension soweit anodisch aufgetragen wurde, daß die der Endosmōse unterliegenden Immunglobuline soweit zur Kathode wanderten, daß sie vom entsprechenden Poolserums bei dessen Trennung in der 1. Dimenision überwandert wurden und dieses partiell oder total präzipitierten. Die humaneñ Ballastproteine des mononospeżifischen Antiserums wanderten während der beiden aufeinander folgenden Elektrophoresen soweit anodisch, daß nur noch wenige in der 2. Dimension dargestellt wurden (Beispiel in Abb. 7).

Spèzielle Färbungen wie die Perjodsäure-Schiff-Reaktion zum Nachweis der Glykoproteine oder die Reaktion mit Schiff'schem Reagenz nach Ozonisierung zur Darstellung der Lipoproteine ergaben wegen der geringen auf der Folie vorhandenen Proteinmengen keine befriedigende Darstellung. Allein die PeroxidaseReaktionen mit Benzidin führte zur deutlichen Darstellung eines Peaks, der später mit monospezifischem Antiserum als dem Haptoglobin entsprechend identifiziert wurde.

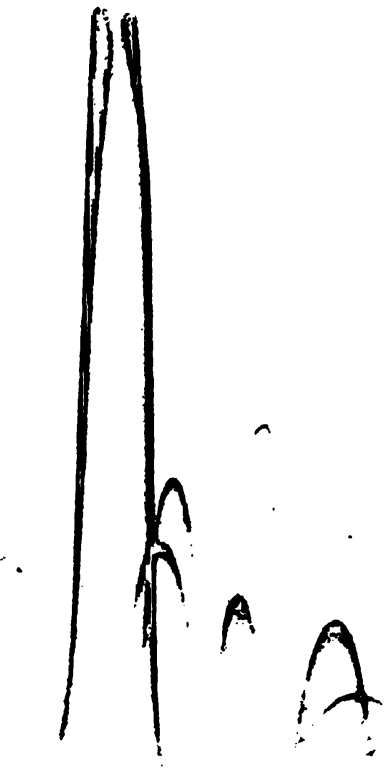

$\oplus$

Abb. 5. Kreuzimmunelektrophorese des Poolserums. (Die scheinbare Doppelbogigkeit der Präzipitate ist aufnahmetechnisch bedingt).

Verwendete monospezifische Antiseren: Anti- $\alpha_{1}$-saures Glycoprotein vom Pferd $^{6}$ ) Anti- $\alpha_{1}$-Lipoprotein vom Kaninchen ${ }^{6}$ ) Anti-Ceruloplasmin vom Kaninchen?) Anti- $\alpha_{2}$-Makroglobulin vom Kaninchen ${ }^{6}$ ) Anti- $\alpha_{2}$-Makroglobulin vom Kaninchen ${ }^{7}$ ) Anti-Haptoglobin vom Kanin chen ${ }^{7}$ ) Anti- $\beta$-Lipoprotein vom Kaninchen ${ }^{7}$ ) Anti-Ig $\dot{G}$ von der Ziege ${ }^{8}$ ) Anti-IgM von der $\mathrm{Ziege}^{8}$ ) Anti-IgA von der Ziege ${ }^{8}$ )

7) Fa. Organon-Teknika, München

8) Fa. Hyland-Travenol, München 


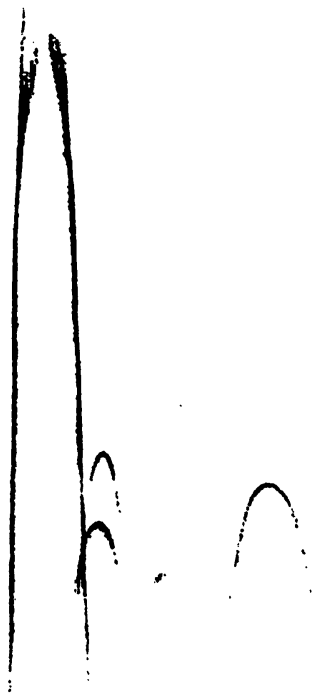

Abb. 6. Identifizierung des Transferrin-Peaks durch Zusatz von Transferrin zum Poolserum: Peak Nr. 18 erhöht. Die beiden zusätzlichen Peaks in der Präalbuminfraktion sind Verunreinigungen des Transferrins.

\section{Ergebnisse und Diskussion}

In Vorversuchen wurde zunächst das günstigste Serum/ Antiserumverhältnis für die Färbung mit Amidoschwarz (25) aufgesucht. Der Äquivalenzbereich wurde dabei für polyvalentes Antihumanserum vom Kaninchen (s.o.) mit $125 \mu \mathrm{l}$ Antiserum pro Folie der zweiten Dimension bei einem Probenauftrag von $2 \mu \mathrm{l} 1+10$ mit Kammerpuffer verdünntem Poolserum gefunden.

Gleichzeitig wurde der Zeitbedarf für die Trennung der zweiten Dimension ermittelt. Bei Laufzeiten von 18-36 Stunden war keine eindeutige Veränderung der Präzipitathöhen nachweisbar. Für die später durchgeführten Versuche zur Identifizierung und die Untersuchung der Reproduzierbarkeit wurde eine Laufzeit von 22-23 Stunden gewählt. Diese Laufzeit entspricht derjenigen der auf Agarose durchgeführten Kreuzimmunelektrophorese $(3,4,8,12,13,22)$. Bei Dünnschicht-AgaroseKreuzimmunelektrophorese wurde allerdings über wesentlich kürzere Laufzeiten von 3-4 Stunden berichtet (9).

Um die je Folie benötigte Antiserummenge weiter zu verringern, wurde schließlich der empfindlicheren Färbung mit Nigrosin der Vorzug gegeben. Hier erwiesen sich $50 \mu$ Antiserum je Folie bei einer Proben-

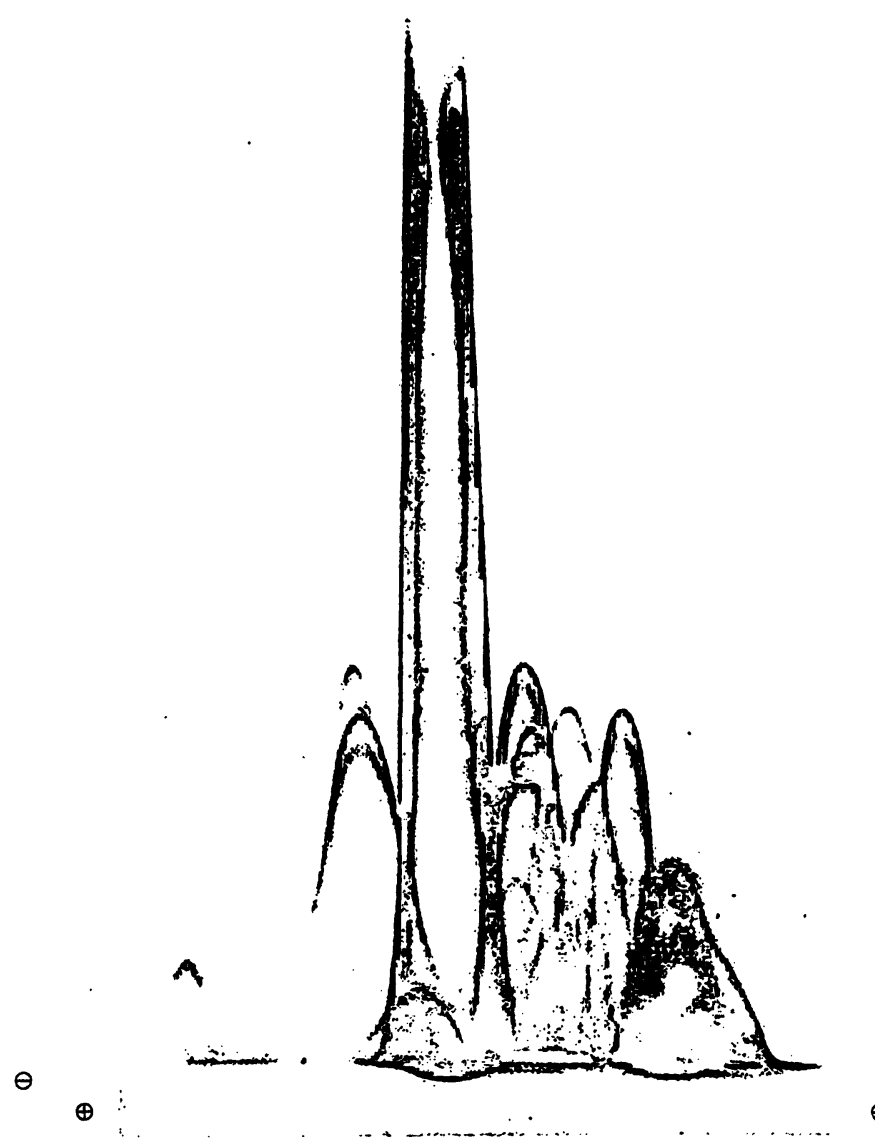

Abb. 7. Identifizierung des Haptoglobin-Peaks mit monospezifischem Anti-Haptoglobin-Serum: Peak Nr. 15 erniedrigt. Humane Ballastproteine des Antiserums als zusätzliche niedrige Peaks in der Präalbumin- bis $\alpha_{1}$-Fraktion. (Die scheinbare Doppelbogigkeit der Präzipitate ist aufnahmetechnisch bedingt).

verdünnung $1+30$ und $2 \mu$ Probenauftrag als ausreichend.

Die Auflage der antiserumhaltigen Folien auf den Celluloseacetatfolien der 1. Dimension ist kritisch, so daß diese nur so breit gewählt wurden, daß eine ausreichende Übersichtlichkeit gesichert ist. Es ist zu erwarten, daß Modifikationen des angegebenen Prinzips, bei denen mit fest eingespannten Folien gearbeitet wird, längere Trennungen mit noch besserer Übersichtlichkeit erlauben werden. Dabei dürfte dem geringen Antiserumverbrauch noch größere Bedeutung zukommen.

Es erwies sich als vorteilhaft, die Folien der 2. Dimension nur jeweils in der Mitte abzustützen. Dies führte zu einem leichten Durchbiegen der Folien. Ein Nachteil für die Trennung ergab sich daraus nicht.

Die Folie der 1 . Dimension ist beiderseits $3 \mathrm{~mm}$ breiter als die aufgelegte, antiserumhaltige Folie. Daraus ergibt sich ein konvergierender Feldlinienverlauf auf der Folie der 1. Dimension während der Trennung der 2. Dimension, der zu einer geringfügigen Verschiebung der jeweils schnellsten bzw. langsamsten Proteine zur Mitte führt. Auf der antiserumhaltigen Folie ist der Feldlinienverlauf parallel. 


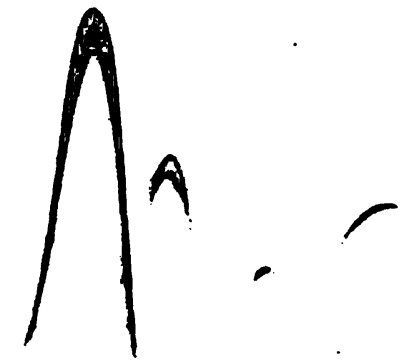

$\oplus$

Abb. 8. Patientenserum. Alkohol-toxische Lebercirrhose. Abweichend von den übrigen Versuchen wurde die Probe $1+60$ verdünnt, um auch für die $\gamma$-Globuline den Äquivalenzbereich einzuhalten. Ein direkter Verg leich mit den Peakhöhen des Poolserums ist daher nicht möglich. Eine ausgeprägte relative Erniedrigung des Albumin- und Transferrin-Peaks bei Höhen- und Breitenzunahme der Immunglobulin-Peaks ist jedoch abzulesen.

Mit Kreuzimmunelektrophoresen, bei denen mit Agarose und nicht vorbehandelten Proben gearbeitet wird, ist eine befriedigende, simultane Darstellung der Immunglobuline wegen der Endosmose nicht zu erreichen $(3,9,30)$. Durch Carbamylierung (5) oder Behandlung des zu trennenden Materials mit $\beta$-Propiolacton (4) konnte eine gute Darstellung der Immunglobuline durch die verringerte positive bzw. erhöhte negative Ladung derselben erreicht werden. Obwohl in der hier beschriebenen Versuchsanordnung eine Endosmose nicht zu erwarten ist, reichen nur wenige Präzipitạte bis zur Basislinie - besonders langsam wandernde Proteine erreichen die antiserumhaltige Folie erst, wenn das Antiserum an der Übergangsstelle infolge eigener Elektrophorese schon erheblich verdünnt ist. Hierzu nach Abschluß der übrigen Untèrsuchungen mit Antiseren verschiedener Tierspezies durchgeführte Versuche zeigten, daß Immunglobuline von der Ziege in der CelluloseacetatfolienZonenelektrophorese wesentlich langsamer zur Anode wanderten als Kaninchenimmunglobuline.

Mit der beschriebenen Technik ließen sich 23 Peaks regelmäßig darstellen (Abb. 4, 5). Ëin Beispiel für die klinische Anwendbarkeit gibt Abbildung 8. Die relativ geringe Anzahl der dargestellten Proteine wurde jedoch wegen der damit gewonnenen Übersichtlichkeit nicht als Nachteil angesehen, da lediglich die Durchführbarkeit der Methode anhand ihrer Reproduzierbarkeit untersucht werden sollte. Aus dem gleichen Grunde wurde auf eine absolute oder relative Quantifizierung der dargestellten Proteine verzichtet und nur der Variationskoeffizient der Peakhöhen als Maß für die Reproduzierbarkeit ermittelt (Tab. 1). Pro Tag wurden vier Kreuzimmunelektrophoresen in einer Kammer durchgeführt. Zwei Kreuzimmunelektrophoresen, bei denen lediglich Poolserum aufgetragen wurde, dienten der Untersuchung der Reproduzierbarkeit, die verbleibenden zwei Kreuzimmunelektrophoresen den Versuchen zur Identifizierung der Präzipitate. Entsprechend dieser Versuchsanordnung sind die ermittelten Variationskoeffizienten einerseits größer als die für Agarose-Kreuzimmunelektrophoresen-Serienuntersuchungen $(9,21)$, andererseits kleiner als die für Agarose-Kreuzimmunelektrophorese von Tag zu Tag (19) mitgeteilten Variationskoeffizienten, die sich auf die Messung der Peakhöhe (21), die näherungsweise Berechnung der Präzipitatflächen (9) und auf das Integral unter der Präzipitatlinie beziehen (19). Mit den bisher beschriebenen Methoden der Kreuzimmunelektrophoresen auf Celluloseacetatfolien sind die Variationskoeffizienten nur bedingt vergleichbar, da diese nur für zwei Peaks (17) angegeben wurden, oder durch Vergleich mit einem internen Standard ermittelt wurden (18) - ein Verfahren, das auch bei der vorliegenden Methode zu einer Verbesserung der Variationskoeffizienten führen könnte.

Die Immunglobuline zeigten asymmetrische Peaks, so daß für diese Proteine eine Höhenmessung nicht zulässig war. Doppelbogigkeit, Verziehungen oder Abrundungen der Peaks traten in keinem Fall auf.

Tab. 1. Variationskoeffizient der Peakhöhen der einzelnen Proteine. Numerierung wie in Abb. 4. (Durchschnittlicher Variationskoeffizient bei 17 Proteinen: 9,5\%)

\begin{tabular}{|c|c|c|}
\hline Peak Nr. & Protein, falls identifiziert & $\begin{array}{l}\text { Variations- } \\
\text { koeffizient }(\%) \\
n=20\end{array}$ \\
\hline 1 & $\alpha_{1}$-Lipoprotein & 8,6 \\
\hline 2 & (Präalbumin?) & 5,9 \\
\hline 3 & Albumin & 4,8 \\
\hline 4 & $\alpha_{1}$-Antitrypsin & 5,6 \\
\hline 5 & & 7,8 \\
\hline 6 & & 6,5 \\
\hline 7 & & 8,5 \\
\hline 8 & & 13,4 \\
\hline 9 & Coeruloplasmin & 7,3 \\
\hline 10 & $\alpha_{2}$-Makroglobulin & 7,6 \\
\hline 11 & & 11,5 \\
\hline 12 & & 9,1 \\
\hline 13 & & 14,4 \\
\hline 14 & & 9,1 \\
\hline 15 & Haptoglobin & 8,3 \\
\hline 17 & & 20,6 \\
\hline 18 & Transferrin & 12,5 \\
\hline
\end{tabular}


Bei Anwendung der beschriebenen Technik ergeben sich folgende Vorteile:

1. Geringerer Antiserumverbrauch. Bei größerem Folienformat $(9 \times 5,6 \mathrm{~cm}) 50 \mu \mathrm{l}$ Antihumanserum je Folie gegenüber $250 \mu \mathrm{l}$ desselben Antiserums je Platte bei $5 \times 5 \mathrm{~cm}$ Agarosegel-Mikrotechnik.

2. Bis auf die Haltevorrichtung in der 2. Dimension ist keine weitere Ausrüstung erforderlich.

3. Geringerer Zeitbedarf. Das Entfernen nicht präzipitierter Proteine, Färben, Auswaschen nicht gebundener Farbe und Trocknen der Platten sind in der AgaroseTechnik zeitraubend, so daß sich hier ein Zeitgewinn von einem Tag bis zu mehreren Tagen ergibt.

\section{Literatur}

1. Grabar, P. \& Williams, C. A. (1953), Biochim. Biophys. Acta 10,193-194.

2. Kindler, U. \& Koch, D. (1970), Dtsch. Med. Wochenschr. 95, $1152-1154$.

3. Clarke, H. G. M. \& Freeman, T. (1968), Clin. Sci. 35, 403-413.

4. Stephan, W. \& Frahm, U. (1971), dieses J. 9, 224-228.

5. Markowski, B. (1973), Clin. Chim. Acta 44, 319-325.

6. Ressler, N. (1960), Clin. Chim. Acta 5, 795-800.

7. Laurell, C.-B. (1965), Analyt. Biochem. 10, 358-361.

8. Weeke, B. (1968), Scand. J. Clin. Lab. Invest. 21, 351-354.

9. Firestone, H. J. \& Aronson, S. B. (1969), Amer. J. Clin. Pathol. 52, 615-622.

10. Kröll, J. (1969), Scand. J. Clin. Lab. Invest. 24, 55-60.

11. Weeke, B. (1970), Scand. J. Clin. Lab. Invest. 25, 161-163.

12. Weeke, B. (1970), Scand. J. Clin. Lab. Invest. 25, 269-275.

13. Stephan, W. \& Frahm, U. (1970), dieses J. 8, 391-393.

14. Raisys, V. \& Arvan, D. A. (1971), Clin. Chem. 17, 745-750.
4. Einfache Durchführung. Die für Agarose-Methoden notwendigen Manipulationen (Herstellen zweier verschiedener Gele, Stanzen, Schneiden, Gießen der Platten) entfallen. Eine gewisse manuelle Geschicklichkeit bleibt jedoch erforderlich.

\section{Dankșagung}

Bei Herrn Dr. W. Herold bedanke ich mich für die Beratung bei der Abfassung der Arbeit, sowie bei Frau Ingrid Taube für die Fotodokumentation.
15. Gay, St., Rosenkranz, M. \& Geiler, G. (1971), Dtsch. Gesundheitsw. 26, 1697-1700.

16. Ganrot, P. O., (1972), Scand. J. Clin. Lab. Invest. 29, Suppl. 124, 39=47.

17. Miller, J. N. \& Mutzelberg, I. D. (1973), J. Chromatogr. 75, $165-168$

18. Pizzolato, M. A. (1973), Clin. Chim. Acta 45, 207-214.

19. Platt, H. S., Sewell, B. M., Feldmann, T. \& Souhami, S. L. (1973); Clin. Chim. Acta 46, 419-429.

20. Schirgel, B. \& Olbert, H. (1974), Z. Inn. Med. 29, 513-517.

21. Schirgel, B. (1974), Z. Inn. Med. 29, 929-932.

22. Groc, W. \& Jendresy, M. (1974), Clin. Chim. Acta 54, $59-69$.

23. Alfonso, E. (1974), Clin. Chim. Acta 54, 123-126.

24. Verbruggen, R. (1975), Clin. Chem. 21, 5-43.

25. Rehfeld, K. H. \& Herold, W. (1976), Med. Lab. 29, 97-109.

26. Glasner, H. (1975), Ärztl. Lab. 232-236.

Dr. K.-H. Rehfeld,

Windscheidstraße 2

1000 Berlin 12 